\title{
O impacto do glaucoma na qualidade de vida: uma revisão sistemática
}

\section{The impact of glaucoma on quality of life: A systematic review}

\author{
Bianca Gomes Queiroz ${ }^{1}$,Lívia Oliveira Delgado Mota²*
}

Como citar esse artigo. Queiroz,

B.G; Mota, L.O.D. O impacto do

glaucoma na qualidade de vida: uma revisão sistemática. Revista de Saúde. 2021 Abr./Jul.; 12 (2): 08-12.

\begin{abstract}
Resumo
O glaucoma é uma neuropatia óptica crônica assimétrica, que causa degeneração lenta e progressiva das células ganglionares da retina. O objetivo desse estudo é avaliar o impacto do glaucoma, analisando os principais domínios da qualidade de vida acometidos pela doença, de modo a auxiliar o profissional de saúde na promoção de uma assistência efetiva aos pacientes glaucomatosos.Foi realizada uma revisão sistemática de literatura com abordagem qualitativa, com as bases Scientific Eletronic Library Online (SciELO), Literatura Latino-Americana e do Caribe em Ciências da Saúde (LILACS) e a National Library of Medicine do PubMed, com artigos em inglês ou português, publicados entre 2015 e 2020, disponíveis por completo em meio eletrônico e estudos do tipo relato de caso, ensaio clínico, ensaio clínico controlado randomizado ou artigo original.Em 33\% dos artigos foram observados que o glaucoma exerce impacto no domínio físico, $31 \%$ abordaram a relevância do ambiente no manejo da doença, $23 \%$ descrevem a relação com problemas psicológicos, enquanto que13\%relatam a influência nas relações sociais. Os indivíduos precisam lidar com limitações psicológicas, ambientais e sociais desde o diagnóstico. Reduzir a taxa de deterioração do campo visual possui efeito significativo na qualidade de vida sendo o diagnóstico tardio o fator de risco mais importante para deficiência visual e cegueira. O estudo evidenciou os efeitos deletérios do glaucoma, ressaltando a necessidade de abordar holisticamente os pacientes glaucomatosos a fim de amenizar o impacto da doença que, além de elevada prevalência, causa cegueira irreversível e afeta diferentes domínios da qualidade de vida.
\end{abstract}

Palavras-chave:Glaucoma; Hipertensão ocular; Qualidade de vida.

\begin{abstract}
Glaucoma is a chronic asymmetric optic neuropathy, which causes slow and progressive degeneration of the retinal ganglion cells. The objective of this study is to assess the impact of glaucoma, analyzing the main domains of quality of life affected by the disease, in order to assist health professionals in promoting effective care for glaucoma patients. A systematic literature review with a qualitative approach was carried out, using the bases Scientific Scientific Electronic Library Online (SciELO), Latin American and Caribbean Literature in Health Sciences (LILACS) and the National Library of Medicine of PubMed, with articles in English or Portuguese, published between 2015 and 2020, available entirely in electronic form and case report, clinical trial, randomized controlled clinical trial or original article. In $33 \%$ of the articles, it was observed that glaucoma has an impact on the physical domain, 31\% addressed the relevance of the environment in the management of the disease, $23 \%$ described the relationship with psychological problems, while $13 \%$ reported the influence on social relationships. Individuals have to deal with psychological, environmental and social limitations since diagnosis. Reducing the rate of deterioration of the visual field has a significant effect on quality of life, with late diagnosis being the most important risk factor for visual impairment and blindness. The study highlighted the deleterious effects of glaucoma, highlighting the need to holistically address glaucoma patients in order to mitigate the impact of the disease, which, in addition to high prevalence, causes irreversible blindness and affects different domains of quality of life.

Keywords: Glaucoma;Ocular hypertension; Quality of life.
\end{abstract}

\section{Introdução}

O glaucoma é uma neuropatia óptica crônica assimétrica, que ocasionaa degeneração das células ganglionares da retina,cujos axônios formam o nervo óptico e conduzem informações para o córtex visual primário ${ }^{1,2}$. A perda de axônios gera declínio da acuidade visual e perda de campo visual correspondente ${ }^{3-7}$. $\mathrm{Na}$ atualidade, é a principal causa de perda de visão no mundo, sendo um grande problema de saúde pública ${ }^{5,8-}$ ${ }^{11}$.

A prevalência dos casos de glaucoma está se elevando com o aumento de expectativa de vida, notase que uma média de $3 \%$ da população acima de 40 anos apresenta o diagnóstico da doença ${ }^{12-14}$. Estima-se que $12 \%$ da proporção de cegos no mundo tenham o glaucoma como causa da perda da visão ${ }^{3}$. Estimou-se que 80 milhões de pessoas desenvolveriam a doença em $2020^{1,7,11,12}$. Isso é um desafio visto que uma média de 65

Afiliação dos autores:

'Discente do Curso de Medicina da Universidade de Vassouras/UV, Vassouras, Rio de Janeiro, Brasil.ORCID: https://orcid.org/0000-0002-6986-5534

${ }^{2}$ Docente do Curso de Medicina da Universidade de Vassouras/UV, Vassouras, Rio de Janeiro, Brasil. ORCID:https://orcid.org/0000-0001-7147-0171

* Email de correspondência:biancagq@hotmail.com 
a 75\% dos casos da doença não apresentam diagnóstico precoce $3,12,13,15$

Os pacientes com glaucoma sofrem com a redução da qualidade de vida (QV) devido diferentes fatores que vão desde a perda da função visual em si até questões psicossociais e econômicas ${ }^{8}$. Qualidade de Vida é "a percepção de um indivíduo de sua inserção na vida, no contexto da cultura e sistemas de valores em que vive e em relação a seus objetivos, expectativas, padrões e preocupações" ". Sendo assim, a QV é um conceito amplo e complexo, que envolve múltiplos domínios que compreendem aspectos diferentes da vida ${ }^{6}$.

A visão relacionada com a qualidade de vida (VRQOL) diz respeito à satisfação da pessoa com sua função ocular e como sua visão exerce impacto em sua vida diária. O glaucoma apresenta consequência diretas como as limitações impostas pela progressiva perda visual e repercussões indiretas como o elevado custo do tratamento e seus efeitos adversos ${ }^{11}$. Soma-se a isso a alta carga psicológica de receber o diagnóstico de uma doença irreversível, de modo que se pode dizer que o bem-estar do indivíduo tem relação inversa com a doença ${ }^{12}$.

Em 2001,foi criado o "25-Item National Eye Institute Visual Function Questionnaire" (NEIVFQ25) que é o questionário mais usado em pacientes com glaucoma. Uma versão validada em português foi produzida em 2008, sendo o único questionário de visão relatada válido no Brasil. Ele possui 25 perguntas referentes a percepção da função visual, divididas em 12 subescalas, incluindo cinco domínios não visuais (saúde geral, saúde mental, dependência, função social, dificuldades funcionais) e sete domínios visuais (visão geral, visão à distância, visão periférica, capacidade de dirigir, visão de perto, visão de cores e dores oculares $)^{5,7,9,16-19}$.]

Outro questionário é o Glaucoma Qualidade de Vida-15 (GQL-15) que possui fácil administração com 15 questões para entender o efeito da perda da visão funcional. Nota-se que quanto maior a gravidade do glaucoma e maior a duração da doença, menor a acuidade visual do paciente e, consequentemente, pontuações maiores no GQL-15, estimando a influência do glaucoma nas atividades diárias ${ }^{20}$.

O NEIVFQ25 e o GQL-15 são questionários amplamente utilizados para estimar a influência do glaucoma na QV, visto que a progressão da doença tem exercido um grande impacto na satisfação não só do paciente bem como de seus familiares, em especial, se a doença não for diagnosticada e tratada precocemente ${ }^{11,13-}$ 15,17. Além disso, o glaucoma foi considerado o principal causador de baixa visão ${ }^{21}$ e estudos demonstram que ocasionou uma redução de 19,1\% em domínios da $\mathrm{QV}^{9}$, reforçando a necessidade de ampliar o conhecimento a respeito dessa doença e melhorar a abordagem desses pacientes. O objetivo do presente estudo é avaliar o impacto do glaucoma na qualidade de vida do indivíduo, analisando os principais domínios da QV acometidos pela doença, de modo a auxiliar o profissional de saúde na promoção de uma assistência mais efetiva aos pacientes glaucomatosos.

\section{Material e Métodos}

Foi realizada uma revisão sistemática de literatura com abordagem qualitativa sobre o quanto as pessoas com glaucoma com a progressão da doença apresentam limitações em domínios da qualidade de vida. A pesquisa usou como fonte de informações as bases de dados eletrônicas Scientific Eletronic Library Online (SciELO), Literatura Latino-Americana e do Caribe em Ciências da Saúde (LILACS)e a National Library of Medicine do PubMed. A seleção dos descritores foi feita mediante a consulta ao DeCS (Descritores em Ciências da Saúde), usando como termos de procura as palavras: "glaucoma", "ocular hypertension", "hipertensão ocular", "qualidade de vida" e "quality of life". Para a localização dos artigos recorreu-se ao operador booleano "and" para combinação dos descritores.

Após a delimitação do tema, foram selecionados os títulos científicos que continham como critérios de inclusão: o ano de publicação restrito a um período de cinco anos (2015-2020), escritos nos idiomas: inglês ou português, estando disponíveis por completo em meio eletrônico e o tipo de desenho de estudos do tipo relato de caso, ensaio clínico, ensaio clínico controlado randomizado ou artigo original.

Em seguida, foi usado como critério de exclusão o nível de evidência dos artigos, mantendo apenas os artigos níveis A e B de evidência, excluindo também todos os artigos duplicados. Por fim, realizou-se a análise qualitativa das informações encontradas por meio da leitura integral dos artigos.

\section{Resultados}

Inicialmente, foram identificados 714 artigos potencialmente elegíveis. Desse total, foram selecionados os artigos que atenderam ao seguinte critério de inclusão: estarem publicados nos últimos 5 anos, totalizando 245 artigos $(\mathrm{PubMed}=243$, LILACS $=$ 1, SciELO =1).Em seguida, selecionou-se apenas artigos dos tipos relato de caso, ensaio clínico, ensaio clínico controlado randomizado ou artigo original, totalizando 240artigos (PubMed= 240, LILACS $=0$, SciELO $=0)$. Desse total, foram selecionados, os artigos em inglês ou português, totalizando artigos 224 (PubMed= 224; $\mathrm{LILACS}=0$; $\mathrm{SciELO}=0$ ). Desses, 116 artigos estavam disponíveis completos em meio eletrônico para a leitura do resumo.Após a análise dos resumos, foram 
excluídos todos aqueles que não se adequaram ao tema ou que estavam duplicados, restando um total de 31 artigos, conforme a Figura 1.

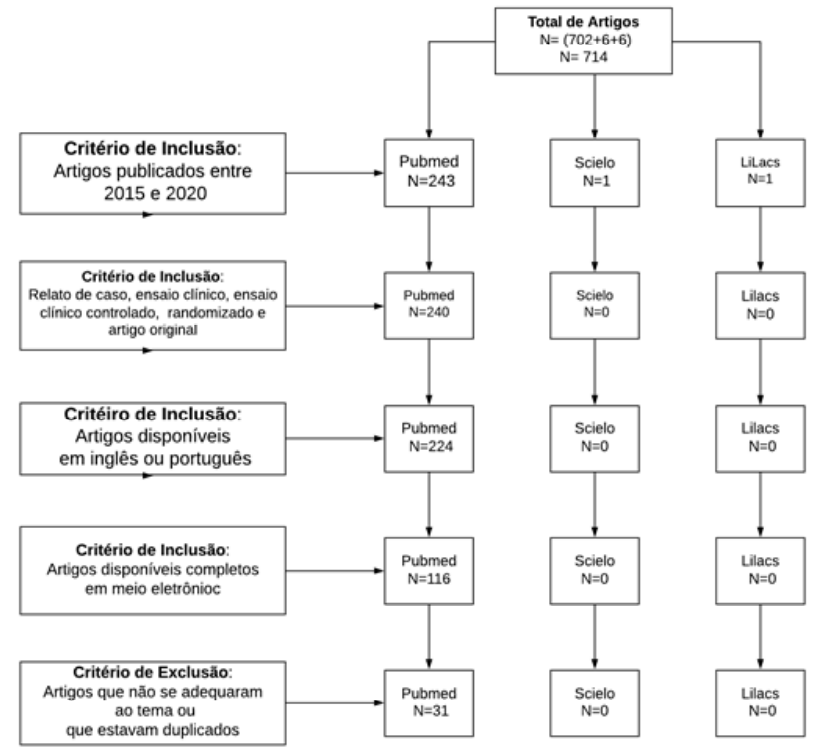

Figura 1. Seleção dos artigos.

Fonte: Elaborado pela autora (2020).

Os 31 artigos foram divididos em categorias de acordo com o domínio da qualidade de vida abordado. Em 33\% dos artigosforam observados que o glaucoma exerce uma grande influência no domínio físico do indivíduo. Entre os trabalhos, 31\% abordaram a relevância do meio ambiente no manejo da doença.

Além disso, 23\% dos estudos descrevem a relação da doença com problemas psicológicos, enquanto que $13 \%$ relatama influência do glaucoma nas relações sociais.As categorias dos estudos estão descritas na Tabela 1 e na Figura 2.

\section{Domínios}

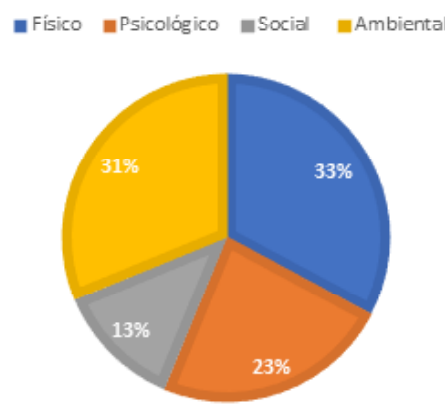

Figura 2. Gráfico representando as porcentagens dos domínios. Fonte: Elaborado pela autora (2020)
Tabela 1. Divisão dos artigos com base nos Domínios Abordados.

\begin{tabular}{l|l|l}
\hline \multicolumn{1}{c|}{ AUTOR } & ANO & Domínios Abordados \\
\hline Ejiakor I & 2019 & Físico, Psicológico, Social, Ambiental \\
\hline Machado LF & 2019 & Físico, Psicológico, Ambiental \\
\hline Yang L & $2019 \mathrm{a}$ & Físico, Ambiental \\
\hline Fraenkel A & 2019 & Físico, Ambiental \\
\hline Shakarchi A & 2019 & Físico, Ambiental \\
\hline Yang L & $2019 \mathrm{~b}$ & Físico \\
\hline Musch DC & 2019 & Psicológico \\
\hline Chun YS & 2019 & Ambiental \\
\hline Fiscella R & 2018 & Ambiental \\
\hline Runjić T & 2018 & Físico, Psicológico, Social, Ambiental \\
\hline Khanna CL & 2018 & Físico, Psicológico \\
\hline Adachi S & 2018 & Físico, Psicológico \\
\hline Rulli E & 2018 & Físico, Ambiental \\
\hline Bieri RAJM & 2018 & Físico, Ambiental \\
\hline Kumar S & 2018 & Físico \\
\hline Bengtsson B & 2018 & Ambiental \\
\hline Sleatha B & 2017 & Físico, Psicológico, Social, Ambiental \\
\hline De Moraes CG & 2017 & Físico, Psicológico, Social, Ambiental \\
\hline Ayele FA & 2017 & Físico, Ambiental \\
\hline Peléié G & 2017 & Psicológico, Ambiental \\
\hline Prager AJ & 2017 & Físico \\
\hline Kuo YS & 2017 & Ambiental \\
\hline Hirooka K & 2017 & Ambiental \\
\hline Lazcano-Gomez G & 2016 & Físico, Psicológico, Social, Ambiental \\
\hline Hirooka K & 2016 & Físico, Psicológico, Social \\
\hline Jung KI & 2016 & Psicológico \\
\hline Bailey LA & 2016 & Físico, Psicológico, Social, Ambiental \\
\hline Alqudah A & 2016 & Físico, Psicológico, Ambiental \\
\hline & & \multicolumn{1}{c}{ Fonte: Elaborado pela autora (2020). } \\
\hline
\end{tabular}

Ao analisar a qualidade de vida é fundamental uma boa compreensão das queixas do indivíduo. Diante disso, foram analisadas as principais dificuldades em cada domínio para possibilitar uma melhor abordagem ao paciente. As duas principais dificuldades dentro de cada domínio estão exibidas na Tabela 2.

Tabela 2. Principais queixas de acordo com o domínio.

\begin{tabular}{l|l}
\hline Domínio & Queixas \\
\hline Físico & Atividades de Vida Diária e Mobilidade \\
\hline Ambiental & Transporte e Cuidados em Saúde \\
\hline Psicológico & Sintomas Negativos e Auto estima \\
\hline Social & Suporte de Pessoas \\
\hline
\end{tabular}




\section{Discussão}

O impacto do glaucoma na QV tem sido amplamente discutido nas últimas décadas. A progressão da doença afeta a QV devido à dificuldade para realizar atividades, dependência familiar e limitações no trabalho, além do uso prolongado de medicamentos ${ }^{5,13}$. A redução dos escores de QV é mais significativa em fases mais tardias da doença, mas o bem-estar do paciente também fica comprometido no início da doença. Sendo assim, os indivíduos precisam lidar com as limitações psicológicas, ambientais e sociais desde o diagnóstico ${ }^{4,9}$.

Muitos pacientes glaucomatosos com grande comprometimento apresentam dificuldade em manter a independência no trabalho. Preservar a acuidade visual permite reduzir o impacto no domínio físico, o que ajuda a manter os índices de $\mathrm{QV}^{8}$.

Além de gastos indiretos com a ausência do trabalho, o tratamento da doença envolve gastos diretos com consultas de rotina,exames, cuidadores, medicamentos, de modo que o impacto econômico é significativo ${ }^{22}$. O aumento de despesas chega ao ponto de 20 a $50 \%$ do salário mensal a ser usado com o tratamento ${ }^{10}$.A busca pelo melhor custo-benefício é essencial, uma vez que o glaucoma gera limitações econômicas pelos elevados custos para a família e para o paciente e, à medida que o diagnóstico é postergado, maiores são os recursos necessários para sustentar um tratamento que retarde a progressão da doença ${ }^{10-12,15,20}$.

A necessidade de apoio da família e de cuidadores para a realização de atividades de vida diárias (AVDs) é notória ${ }^{2}$. O glaucoma vai progressivamente reduzindo o campo visual o que acarreta mais dificuldade para enxergar os objetos com clareza, limitando a realização das AVDs, como se alimentar, se vestir, ler, escrever e até tomar banho ${ }^{5,7,11,19,23}$. A falta de autonomia aumenta com o avanço da severidade da doença, de modo que quanto maior deficiência visual, mais dependência de outras pessoas, afetando as relações sociais de pacientes glaucomatosos ${ }^{24,25}$.

O glaucoma ocasiona um grande impacto psicológico, não apenas pela perda da independência e dificuldade de adaptação, mas também pela redução de escores de bem-estar emocional conforme a doença evolui ${ }^{6,21,26-28}$. Os traumas psicológicos relacionados a perda visual são diversos: maiores níveis de estresse, distúrbios do sono, solidão, depressão, ansiedade ${ }^{3,12}$. Os pacientes exibem maior tendência a depressão e, com a severidade da doença, os sintomas depressivos ficam mais proeminentes.

A percepção do paciente sobre a doença influencia diretamente na adesão ao tratamento. Indivíduos com baixa escolaridade apresentaram menor entendimento sobre a doença e menor adesão medicamentosa ${ }^{6,19,26}$. A má adesão envolve diversos fatores como as despesas e as dificuldades para entender o esquema de administração e dosagens dos remédios, o que piora a pressão intraocular (PIO), afetando a $\mathrm{QV}^{13}$. O tratamento precoce promoveu um impacto positivo no curso da doença, reduzindo o número de visitas ao oftalmologista e os custos com o tratamento ${ }^{18}$.

Algumas terapias para redução da PIO podem estar associadas a efeitos adversos leves, moderados ou graves ${ }^{14}$. Orientar o paciente a respeito de possíveis efeitos colaterais e sobre as consequências graves da má aderência ao tratamento é fundamental para garantir o bem-estar do indivíduo. Diante disso, os profissionais de saúde precisam monitorar com regularidade a $\mathrm{QV}$ do paciente glaucomatoso, aplicando questionários e discutindo estratégias sobre como melhorar a $\mathrm{QV}^{25,29}$.

$\mathrm{O}$ domínio do meio ambiente é afetado principalmente na habilidade de transporte. A capacidade de dirigir é afetada pela dificuldade de adaptação à diferentes luminosidades,em especial no período da noite pelo prejuízo na visão noturna e em dias nublados $2,15,19,24,26$.

Uma estratégia simples e efetiva para prevenir a perda visual é a suspeita diagnóstica, uma vez que o diagnóstico tardio é o fator mais importante para deficiência visual e cegueira ${ }^{30}$. A criação de planos para o diagnóstico precoce é imprescindível, sendo importante rastrear a doença em maiores de 40 anos $^{15,20,29}$. O paciente com glaucoma deve receber tratamento durante toda a sua vida útil e a comunicação entre o médico e o paciente se torna essencial nesse processo ${ }^{8,14,31,32}$.

\section{Considerações finais}

O estudo evidenciou os efeitos deletérios do glaucoma na $\mathrm{QV}$, ressaltando a necessidade de abordar holisticamente os pacientes glaucomatosos a fim de amenizar o impacto da doença que, além de elevada prevalência, causa cegueira irreversível e afeta diferentes domínios da QV. É fundamental a criação de políticas de saúde para a redução de custos, assim como intervenções que facilitem a realização de atividades do cotidiano com modificações no estilo de vidapara reduzir a dependência financeira e social do paciente.

A reabilitação visual e o manejo das restrições de mobilidade necessitam de equipe multidisciplinar.É importante abordar também a aderência e a tolerância ao tratamento, uma vez que postergar o tratamento eleva os riscos de progressão da doença e, consequentemente, reduz os índices de QV.

Limitações do estudo incluem a falta de informações sobre o assunto, devido a existência de poucos artigos que explorem diretamente as consequências do glaucoma em diferentes domínios, gerando dificuldade em mensurar as consequências da 
doença na qualidade de vida. O maior desafio atual é o conhecimento da história natural do glaucoma pelos profissionais de saúde, possibilitando a suspeita, o diagnóstico e o tratamento precoce da doença, a fim de garantir uma melhor QV ao indivíduo.

\section{Referências}

1. Adachi S, Yuki K, Awano-Tanabe S, Ono Takeshi, Shiba D, Murata $\mathrm{H}$, et al. Factors associated with developing a fear of falling in subjects with primary openangle glaucoma. BMC Ophthalmol2018;18:39.

2. Ronald AJMB, Frideric LPS, Nomdo MJ. Visual complaints of patients with glaucoma and controls under optimal and extreme luminance conditions. Acta Ophthalmol 2018;96:288-294.

3. Hirooka K, Sato S, Nitta E, Tsujikawa A. The Relationship Between Vision-related Quality of Life and Visual Function in Glaucoma Patients. J Glaucoma2016;25:505-509.

4. Prager AJ, Hood CD, Liebmann JM, De Moraes CG, Al-Aswad L, Yu QI, et al. Association of Glaucoma-Related, Optical Coherence Tomography-Measured Macular Damage with Vision-Related Quality of Life. JAMA Ophthalmol 2017;135(7):783-788.

5. Waisbourd M, Parker S, Ekici F, Martinez P, Murphy R, Scully K, et al. A prospective, longitudinal, observational cohort study examining how glaucoma affects quality of life and visually-related function over 4 years: design and methodology. BMC Ophthalmol2015; 15: 91.

6. Runjić T, Lauš KN, Vatavuk Z. Efect of diferente visual impairment levels on the quality of life in glaucoma patients. Acta Clin Croat 2018; $57: 243-250$

7. Machado LF, Kawamuro M, Portela RC, Fares NT, Bergamo V, De Souza LM, et al. Factors associated with vision-related quality of life in Brazilian patients with glaucoma. Arq Bras Oftalmol 2019;82(6):463-7.

8. Chun YS, Sung KR, Park CK, Kim HK, Yoo C, Kim YY,et al. Factors influencing vision-related quality of life according to glaucoma severity. Acta Ophthalmol 2019; 97: e216-e224.

9. Ejiakor I, Achigbu E, Onyia O, Edema O, Florence NU. Impact of Visual Impairment and Blindness on Quality of Life of Patients in Owerri, Imo State, Nigeria. Middle East Afr J Ophthalmol2019;26:127-32.

10. Hirooka K, Nitta E, Ukegawa K, Tsujikawa A. Vision-related quality of lifefollowing glaucoma filtration surgery. BMC Ophthalmol2017; 17: 66.

11. Rulli E, Quaranta L, Riva I, Poli D, Hollander L, Galli F, et al. Visual field loss and vision-related quality of life in the Italian Primary Open Angle Glaucoma Study. Scientific Reports2018; 8:619.

12. Lazcano-Gomez G, Ramos-Cadena MLA, Torres-Tamayo M, Oteyza AH, Turati-Acosta M, Jimenez-Román J. Cost of glaucoma treatment in a developing country over a 5-year period. Medicine2016;95:47(e5341).

13. Pelěiè G, Ljubiěié R, Baraé J, Biuk D, Rogoié V. Glaucoma, depression and quality of life: multiple comorbidities, multiple assessments and multidisciplinar plan treatment. PsychiatriaDanubina2017; 29(3): 351359.

14. De Moraes CG, Liebmanna JM, Levin LA. Detection and measurement of clinically meaningful visual field progression in clinical trials for glaucoma. Prog Retin Eye Res 2017; 56: 107-147.

15. Ayele FA, Zeraye B, Assefa Y, Legesse K, Azale T, Burton MJ. The impact of glaucoma on quality of life in Ethiopia: a case-control study. BMC Ophthalmol2017; 17:248.

16. Biblioteca Virtual emSaúde [homepage na internet]. Qualidade de vidaem 5 passos[acessoem 8 jul 2020]. Disponívelem: https://bvsms.saude. gov.br

17. Yang L, Shi X, Tang X. Associations of subjective and objective clinical outcomes of visual functions with quality of life in Chinese glaucoma patients: a cross-sectional study. BMC Ophthalmol 2019; 19:166.
18. Bengtsson B, Heijl A, Johannesson G, Andersson-Geimer S, Aspberg J, Lindén C. The Glaucoma Intensive Treatment Study (GITS), a randomized clinical trial: design, methodology and baseline data. Acta Ophthalmol 2018; 96: $557-566$

19. KuoYS,LiuCJ,ChengHC,ChenMJ,ChenWT,Ko YC. Impact of socioeconomic status on vision-related quality of life in primary open-angle glaucoma. Eye2017; 31: 1480-1487.

20. Kumar S, Ichhpujani P, Singh R, Thakur S, Sharma M, Nagpal N. The impact of primary open-angle glaucoma: Quality of life in Indian patients.Indian J Ophthalmol 2018;66:416-419.

21. Peters D, Heijl A, Brenner L, Bengtsson B. Visual impairment and vision-related quality of life in the Early Manifest Glaucoma Trial after 20 years of follow-up. Acta Ophthalmol. 2015; 93: 745-752.

22. Guedes RAP, Guedes VMP, Gomes CEM, Chaoubah A. Maximizing cost-effectiveness by adjusting treatment strategy according to glaucoma severity. Medicine2016; 95:52.

23. Yang L, Tang X. Bilateral asymmetry improved accuracy when assessing glaucomatous vision-related quality of life impairment. Medicine2019; 98:45.

24. Shakarchi AF, Mihailovic A, West SK, Friedman DS, Ramulu PY. Vision Parameters Most Important to Functionality in Glaucoma. Invest Ophthalmol Vis Sci2019;60(14):4556-4563.

25. Sleath B, Sayner R, Vitko M, Carpenter DM, Blalock SJ, Muir KW, et al. Glaucoma patient-provider communication about vision quality-oflife. Patient Educ Couns2017; 100(4): 703-709.

26. Fiscella R, Caplan E, Kamble P, Bunniran S, Uribe C, Chandwani $\mathrm{H}$. The Effect of an Educational Intervention on Adherence to Intraocular Pressure-Lowering Medications in a Large Cohort of Older Adults with Glaucoma. J Manag Care Spec Pharm 2018;24(12):1284-94.

27. Bailey LA, Okereke OI, Kawachi I, Cioffi GA, Pasquale LR, Kang JH. Ophthalmic and Glaucoma Treatment Characteristics Associated with Changes in Health-Related Quality of Life Before and After Newly Diagnosed Primary Open-Angle Glaucoma in Nurses Health Study Participants. J Glaucoma2016; 25(3): e220-e228.

28. Khanna CL, Leske DA, Holmes JM. Factors Associated with Health-Related Quality of Life in Medically and Surgically Treated Patients withGlaucom. JAMA Ophthalmol 2018;136(4):348-355.

29. Jung KI, Park CK. Mental Health Status and Quality of Life in Undiagnosed Glaucoma Patients: A Nationwide Population-Based Study. Medicine2016; 95(19):e3523.

30. Alqudah A, Mansberger SL, Gardiner SK, Demirel S. Visionrelated quality of life in glaucoma suspect or early glaucoma patients. J Glaucoma2016 ago; 25(8): 629-633.

31. Musch DC, Niziol LM, Janz NK, Gillespie BW. Trends in and Predictors of Depression among Participants in the Collaborative Initial Glaucoma Treatment Study (CIGTS). Am J Ophthalmol 2019; 197: 128135 .

32. Fraenkel A, Lee GA, Vincent SJ, Vincent RA, Bourne RRA, Shah P. Lessons learned from the development and implementation of a patientreported outcome and experience measure (POEM) in an Australian glaucoma practice. BMC Ophthalmol. 2019; 19:192. 